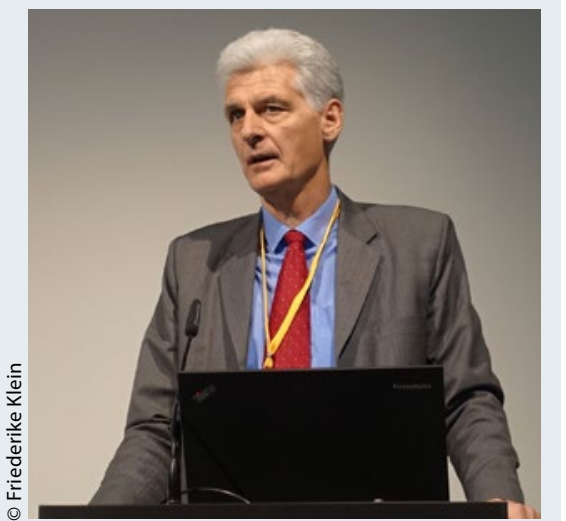

Dr. Rolf Schmachtenberg vom Bundessozialministerium

\section{Bundesteilhabegesetz seit dem 1. Januar 2017 in Kraft}

Am 2. Dezember 2016 verabschiedete der Deutsche Bundestag nach zweiter und dritter Lesung das neue Bundesteilhabegesetz, am 16. Dezember 2016 der Bundesrat. Zum 1. Januar 2017 trat das Gesetz in Kraft. In drei Stufen sollen die Neuerungen umgesetzt werden und durch regelmäßigen Erfahrungsaustausch und begleitende Beobachtungen laufend verbessert werden. Außerdem sind in der Folge noch weitere Änderungen notwendig, beispielsweise im Behindertengleichstellungsgesetz oder im Nationalen Aktionsplan Behinderung. „Für die Liebhaber von Beteiligungsprozessen: Wie haben da noch was", meinte Dr. Rolf Schmachtenberg (im Bild), Leiter der Abteilung V - Teilhabe, Belange behinderter Menschen, soziale Entschädigung, Sozialhilfe im Bundesministerium für Arbeit und Soziales, anlässlich des DGPPN-Kongresses am 24. November 2016.

Das Bundesteilhabegesetz ist keine deutsche Errungenschaft, sondern der Hausaufgabe geschuldet, die die UN-Behindertenrechtskonvention der Bundesregierung gegeben hat. Nun versuchen die Verantwortlichen einen Spagat, betonte Schmachtenberg: Die Verbesserung der Lebenssituation von Menschen mit Behinderung im Lichte dieser Konvention auf der einen, ohne neue Ausgabendynamik in der Eingliederungshilfe auf der anderen Seite. Immerhin: Für Modellprojekte werden vom Bund fünf Jahre lang eine Milliarde Euro zur Verfügung gestellt.

Friederike Klein

Hauptsymposium 14 „Bundesteilhabegesetz 2017: Was folgt daraus für Menschen mit schweren psychischen Erkrankungen?" DGPPN-Kongress 2016, Berlin, 24.11.2016 grund von ihr zu bezahlender, nicht so hoch dotierter Verkehrswerte einen Aufkauf in Betracht ziehen. Als Abwehrmittel hiergegen ist eine MVZ-Gründung bestens geeignet.

\section{Urteil des Bundessozialgerichts}

In einschlägigen Fachmedien wurde bereits mehrfach über eine grundlegende Entscheidung des Bundessozialgerichts (BSG) vom 4. Mai 2016 („MVZ-Urteil“) berichtet. Nach $\$ 103$ Absatz 4a SGB V kann ein Vertragsarztsitz in ein MVZ „eingebracht“ werden, indem ein Vertragsarzt in einem gesperrten Planungsbereich auf seine Zulassung verzichtet, „um in einem MVZ tätig zu werden“. In diesem Kontext „kaufen“ MVZ Vertragsarztpraxen auf, indem sie den betreffenden Vertragsarzt eine im Einzelfall hohe Abfindung für den Verkehrswert seiner Vertragsarztpraxis oder seines einhergehenden Praxisanteils an einer BAG bezahlen, wenn der Vertragsarzt künftig in dem MVZ als angestellter Arzt tätig sein wird. In diesem Zusammenhang sind ein Kauf- sowie ein Anstellungsvertrag mit dem künftigen angestellten Arzt im MVZ zu schließen. Nach dem zitierten Urteil des Bundessozialgerichts setzt dieses allerdings für eine rechtsbeständige „Umwandlung“ des Vertragsarztsitzes des in das MVZ als angestellter Arzt eintretenden Vertragsarztes voraus, dass die Tätigkeit des (früheren) Vertragsarztes in dem MVZ für einen Zeitraum von mindestens drei Jahren ausgeübt wird. Nur dann entsteht für das MVZ das wirtschaftlich fundamental wichtige Nachbesetzungsrecht für den Fall des Ausscheidens des betreffenden angestellten Arztes aus dem MVZ nach $\$ 103$ Absatz 4a Satz 3 SGB V. Nach dem Urteil des BSG besteht dieses Nachbesetzungsrecht im Regelfall nicht, wenn der betreffende (frühere) Vertragsarzt vor Ablauf von drei Jahren aus dem MVZ ausscheidet, es sei denn, es kann „nach den Umständen davon ausgegangen werden, dass der ursprünglich zugelassene Arzt zunächst tatsächlich zumindest drei Jahre im MVZ tätig werden wollte, diese Absicht aber aufgrund von Umständen, die ihm zum Zeitpunkt des Verzichts auf die Zulassung noch nicht bekannt waren, nicht mehr realisieren konnte. Dies kann etwa der Fall sein, wenn er erkrankt oder aus zwingenden Gründen seine Berufs- oder Lebensplanung ändern musste“.

Hat im Einzelfall der eintretende (frühere) Vertragsarzt schon im Falle des Eintritts in das MVZ konkrete Pläne für die Beendigung seiner ärztlichen Tätigkeit und/oder führte das MVZ zu diesem Zeitpunkt schon Verhandlungen mit einem an der Nachbesetzung der betroffenen Arztstelle interessierten anderen Arzt, geht das Nachbesetzungsrecht des MVZ bei Austritt des (früheren) Vertragsarztes vor Ablauf des 3-JahresZeitraums nach dem Urteil des BSG unter.

Die Rechtslage des betreffenden Urteils gilt kraft ausdrücklicher Regelung erst für Nachbesetzungen, die sich auf Arztstellen beziehen, denen Umwandlungsanträge nach der Verkündung des Urteils, mithin nach dem 4. Mai 2016 zugrunde liegen. Vereinfacht ausgedrückt gilt das besprochene Urteil nicht für Altfälle, sondern lediglich für Neufälle. In Kaufverträgen mit in ein MVZ als angestellte Ärzte eintretenden früheren Vertragsärzten, die ihren Vertragsarztsitz in das MVZ einbringen, sind daher Regelungen vorzusehen, dass die Tätigkeit des betreffenden Vertragsarztes den Zeitraum von drei Jahren überschreiten muss. Rechtstechnisch kann hier beispielsweise mit Bedingungsklauseln gearbeitet werden. Auch können Regelungen getroffen werden, dass ein Kaufpreis ganz oder teilweise bei vorzeitigem Ausscheiden des in das MVZ eintretenden (früheren) Vertragsarztes zurückzubezahlen ist. Es muss auch an weitere Absicherungskonstruktionen für das MVZ gedacht werden. In Anstellungsverträgen mit in das MVZ als angestellte Ärzte eintretenden (früheren) Vertragsärzten sind Befristungsregelungen mit einer Dauer von mindestens drei Jahren vorzusehen. Aufgrund der brandneuen Rechtslage bedürfen Einzelheiten der sorgfältigen Prüfung und vertraglichen Gestaltung durch einen erfahrenen anwaltlichen Berater.

\section{Dr. Alexander Böck}

Rechtsanwalt

Herdweg 62, 70174 Stuttgart

E-Mail: boeck@boeck-law.de

www.boeck-law.de 